\title{
Design of a Low Phase Noise VCO for Rubidium Atomic Frequency Standard
}

\author{
Qingyun $\mathrm{Ju}^{1}$, Xinwei $\mathrm{Li}^{1}$, Lei $\mathrm{Ji}^{1}$, Liang $\mathrm{Tang}^{2}$ and Donghai Qiao ${ }^{2}$ \\ ${ }^{1}$ School of Electronic and Information Engineering, Soochow University, 215000 Suzhou, China \\ ${ }^{2}$ Institute of Acoustics, Chinese Academy of Sciences, 100000 Beijing, China
}

\begin{abstract}
Compared to the size and the power consumption of the traditional atomic clocks, the ones based on coherent population trapping (CPT) can provide improvement by two orders of magnitude in both aspects above, making them needed urgently in many fields. Among different CPT atomic clocks, the one made with the rubidium atom are used widely, and their operating performance largely depends on its internal voltage-controlled oscillator (VCO) which is used to provide a proper microwave signal. Based on this, a small size and low phase noise $3.035 \mathrm{GHz}$ VCO is designed with a modified Clapp circuit topology using low-cost surface-mount components, including a coaxial resonant (COAX) with high quality factor. The designed VCO is simulated and optimized with the combined use of the negative resistance analysis method and the transmission analysis with a virtual-ground. In order to obtain the desired results, different values of key capacitor elements are tried according to their concrete influences on the VCO during the process of the circuit tuning. The test results show that the phase noises of the VCO are $-60.49 \mathrm{dBc} / \mathrm{Hz} @ 300 \mathrm{~Hz},-73.08 \mathrm{dBc} / \mathrm{Hz} @ 1 \mathrm{KHz}$ and $-97.48 \mathrm{dBc} / \mathrm{Hz} @ 10 \mathrm{KHz}$, the output power is $-1.13 \mathrm{dBm}$ and the voltage-controlled tuning sensitivity is about $12 \mathrm{MHz} / \mathrm{V}$.
\end{abstract}

\section{Introduction}

With the progress of the society and the development of the science and technology, there is a growing demand for timing tools with small size, high precision and low power consumption in many fields, particularly in the military industry and the precision measurement field. Atomic clocks based on CPT can meet all the specs well [1] and they can be realized with different atoms among which the rubidium atom is most widely used. According to the operating mechanism of the CPT atomic clocks, their performance largely depends on the internal VCO [2]. The frequency of the $\mathrm{VCO}$ is designed to be equal to or half of the atomic ground state hyperfine splitting frequency to modulate the laser effectively [2]. An appropriate frequency tuning ability is also needed to compensate for the frequency drift caused by component tolerances, temperature changes and other factors [3]. In this paper, a low phase noise $3.035 \mathrm{GHz} \mathrm{VCO}$ based on a COAX with high quality factor is proposed, which has the potential to be used in ${ }^{85} \mathrm{Rb} \mathrm{CPT}$ atomic clocks.

In order to design the VCO, the overall circuit can be designed firstly with the negative resistance analysis method which can make the oscillator start up fast, and with the use of the virtual-ground technology, the circuit can be evolved into a form that a gain circuit in series with a resonance circuit. The oscillation frequency and the gain margin of the circuit can be determined according to the Nyquist criterion [4]. Parameters of the circuit are optimized by observing the magnitude and phase responses of the open-loop gain to obtain better performance of the designed VCO.

\section{The structure and design method}

\subsection{The structure of the VCO}

The VCO circuit is designed with a Clapper structure including a resonance circuit, a gain circuit, a voltagecontrolled bias circuit and an output isolation circuit. The operating frequency is determined by the resonance circuit which is composed of a COAX with high quality factor and a varactor with good linearity. The gain circuit is not only used to provide a suitable gain for the oscillator circuit, but also to maintain the stable oscillation. And value of the gain should be large enough to achieve oscillation but be low enough to prevent the transistor from strong compression and to reduce oscillations at higher harmonics. The voltage-controlled bias circuit is used to control the reverse bias voltage of the varactor to realize the adjustment of the oscillation frequency. The output isolation circuit is used to isolate the VCO and the test instruments to reduce the bad influences of the impedance mismatch and the load pull. The attenuation value can be designed based on the needed output power of the VCO whose principle frame diagram is shown in Fig. 1. 


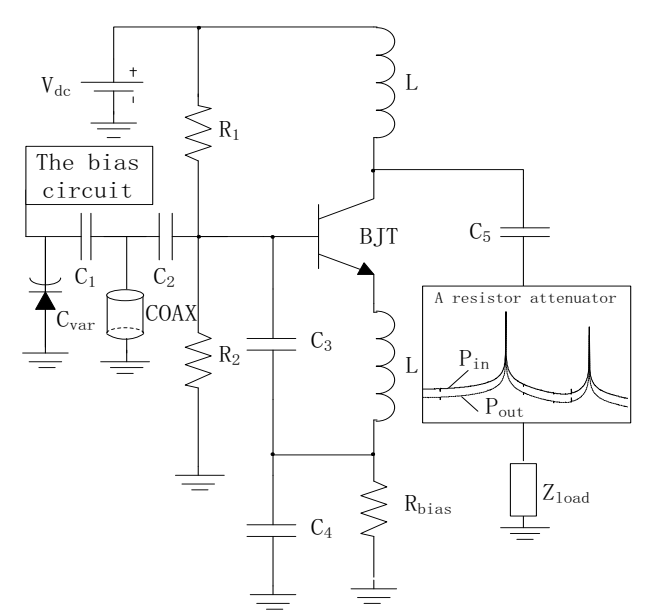

Figure 1. The principle frame diagram of VCO

\subsection{Theories about the used design methods}

At the beginning, the VCO is designed with the negative resistance analysis method which needs to satisfy (1),

$$
Z_{T}=R_{T}+j X_{T}=0
$$

where $Z_{T}$ is the total impedance of the circuit, $R_{T}$ and $X_{T}$ are the real part and the imaginary part of $Z_{T}$, respectively. And they can be expressed in (2) and (3),

$$
\begin{aligned}
& R_{T}=R_{S}+R_{L}=0 \\
& X_{T}=X_{S}+X_{L}=0
\end{aligned}
$$

where $R_{S} 、 X_{S} 、 R_{L} 、 X_{L}$ are the real and imaginary parts of the source impedance and load impedance, respectively. For a passive load, the real part of the impedance is always positive, so the active device should present an appropriate negative resistance when the real part of the total impedance is equal to 0 . The condition in (2) mainly determines whether the oscillator can start up. When the oscillation occurs, the total resistance needs to be less than 0 , and then there is a continuous growing current corresponding to the frequency in the circuit. As the current increases, the absolute value of the total resistance decreases gradually until the current of the circuit has been a stable value, meaning that the total resistance of the circuit has been equal to 0 . On the other hand, the condition in (3) mainly determines the oscillation frequency of the circuit.

However, the conditions stated above are not sufficient to ensure the circuit can oscillate steadily. The stable oscillation also requires that the frequency disturbances and current disturbances caused by different factors can be gradually reduced to none. The frequency disturbances can be adjusted by the control voltage of the VCO, while the current or amplitude disturbances need to present an exponential decay. In this case, the total impedance of the circuit should meet the Kurokawa condition [5] in (4).

$$
\frac{\partial R_{T}}{\partial I} \frac{\partial X_{T}}{\partial \omega}-\frac{\partial X_{T}}{\partial I} \frac{\partial R_{T}}{\partial \omega}>0
$$

For a passive load, the real and imaginary parts of the total impedance of the VCO usually meet the conditions that $\partial R_{L} / \partial I=\partial X_{L} / \partial I=\partial R_{L} / \partial \omega=0$, meaning that (4) can be expressed as (5).

$$
\frac{\partial R_{S}}{\partial I} \frac{\partial}{\partial \omega}\left(X_{S}+X_{L}\right)-\frac{\partial X_{S}}{\partial I} \frac{\partial R_{S}}{\partial \omega}>0
$$

Usually, $\partial R_{S} / \partial I>0$ can be satisfied in the start-up process, so (5) can be well met if $\partial\left(X_{S}+X_{L}\right) / \partial \omega>>0$, indicating that the total reactance which determines the oscillation frequency has a steep phase slope near the resonant frequency. This implies that a high quality factor circuit will result in maximum oscillator stability. In this paper, a COAX with high unloaded quality factor of about 450 is chosen to provide strong guarantee for good performance of the designed VCO.

However, on the basis of the negative resistance analysis method, the total impendence of the circuit is equal to 0 when the oscillation is stable, meaning that the quality factor is infinite. This situation is obviously impossible so that the phase noise evaluated with the Leeson formula relating to quality factor of the circuit is meaningless. The Leeson formula is shown in (6) [3-4],

$$
L(\Delta f)=10 \lg \left\{\frac{F k T}{2 P_{i n}}\left[1+\left(\frac{f_{0}}{2 \Delta f Q_{L}}\right)^{2}\left(1+\frac{f_{c}}{\Delta f}\right)\right]\right\}
$$

where $\Delta f$ is the frequency offset from the carrier center frequency $f_{0}, L(\Delta f)$ is the ratio of the sideband power in a $1 \mathrm{~Hz}$ bandwidth at the offset of $\Delta f$ to the total output power generated by the oscillator in $\mathrm{dBc} / \mathrm{Hz}, F$ is the large signal noise figure of the active device in $\mathrm{dB}, k$ is the Boltzmann's constant, $T$ is the equivalent noise temperature in $\mathrm{K}, P_{i n}$ is the signal power at oscillator input in $\mathrm{dBm}, Q_{L}$ is the loaded quality factor and $f_{c}$ is the flicker corner frequency of the active device.

In addition, the gain of the circuit cannot be observed directly with the negative resistance analysis method. The circuit in Fig. 1 can be evolved into a two port circuit in which a gain circuit and a resonance circuit are connected in a series form using the virtual-ground technology [6]. In this case, the amplitude and phase of the open-loop gain can be observed by the S-parameters, the resonant frequency of the circuit can be obtained by the zero phase crossings and the loaded quality factor of the circuit can be roughly estimated according to the group delay.

\section{Circuit simulation}

\subsection{The equivalent model of the COAX}

Normally, the oscillation frequency of the VCO designed with a COAX is lower than the self-resonant frequency of the COAX which can be equivalent to an inductor with high quality factor on this occasion. According to the impedance characteristics of the COAX, the impedance changes fastest near the self-resonant frequency so that 
the frequency tuning range of the circuit will be restricted. For example, when the resonant frequency of the circuit is increased by reducing the capacitance of the varactor in the vicinity of the self-resonant frequency, the equivalent inductor of the COAX will be increased in the form of tangent function, then the resonant frequency of the circuit is decreased apparently, which is opposite to the adjusting direction of the varactor, and vice versa [3]. Therefore, the self-resonant frequency of the COAX needs to be properly higher than the operating frequency. In this design, the customized COAX has a self-resonant frequency of $3.104 \mathrm{GHz}$ and an unloaded quality factor of 458. And a large enough frequency tuning range and a high loaded quality factor can be expected at the desired frequency of $3.035 \mathrm{GHz}$.

The resonance circuit composed by the COAX and a coupling capacitor is shown in Fig. 2. The TLPSC is the model of the used COAX in ADS software and its parameters can be obtained via the manufacturer. The L1 is the pin inductor of the COAX and its value is $0.6 \mathrm{nH}$ according to its data sheet. The $\mathrm{C} 1$ is the coupling capacitor and it constitutes a resonance circuit together with the COAX. The Term1 represents the input impedance of the transistor under a given bias voltage. In the simulation process, the parameters of the COAX need to be modulated slightly in the range of tolerance in order to realize resonance at the frequency of $3.035 \mathrm{GHz}$.

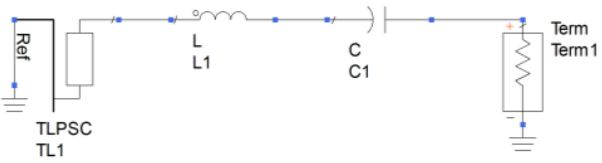

Figure 2. The equivalent model of the COAX.

Fig. 3 shows the S-parameter simulation results of the circuit. As can be seen, the circuit achieves resonance at $3.035 \mathrm{GHz}$. And then the VCO can be designed based on the resonance circuit in Fig. 2.

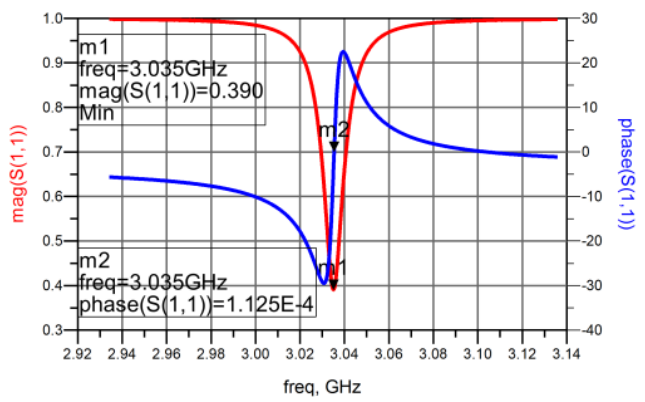

Figure 3. The simulation results of the resonance circuit.

\subsection{Design with the negative resistance analysis method}

The resonance circuit with high quality factor in Fig. 2 is connected to the base electrode of the transistor which has a high gain, an appropriate cut-off frequency and a low noise figure near the frequency of $3.035 \mathrm{GHz}$. The chosen super-abrupt junction varactor possesses a low equivalent series resistor, contributing to obtaining a low phase noise. When the VCO is simulated, the varactor is replaced by an equivalent series RLC circuit and lightly coupled into the circuit with a proper capacitor. And then a modified Clapp circuit can be built along with the COAX. A resistor divider circuit is placed at the base electrode of the transistor to provide a proper bias voltage. In order to stabilize the static operation point of the transistor, its emitter electrode is connected with a resistor which can be used as the negative feedback component. The oscillation signal is output using a coupled capacitor after which a resistor attenuator is placed to provide the VCO some isolation from the load. The principle diagram of the VCO is shown in Fig. 4.

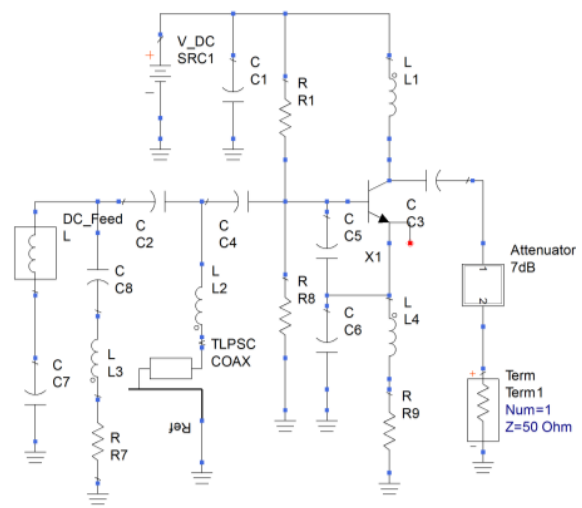

Figure 4. The principle diagram of the VCO.

Based on the negative resistance analysis method, the values of C5, C6 and R9 in Fig. 4 are adjusted and optimized so that the circuit can present a large enough reflection coefficient when seen from the base electrode of the transistor and a proper negative resistance. The simulation result of the reflection coefficient is shown in Fig. 5. It can be seen that the maximum value of the curve is $8.304 \mathrm{~dB}$ achieved at $3.035 \mathrm{GHz}$, indicating that a big enough negative resistance can be expected and the designed circuit can start up near $3.035 \mathrm{GHz}$.

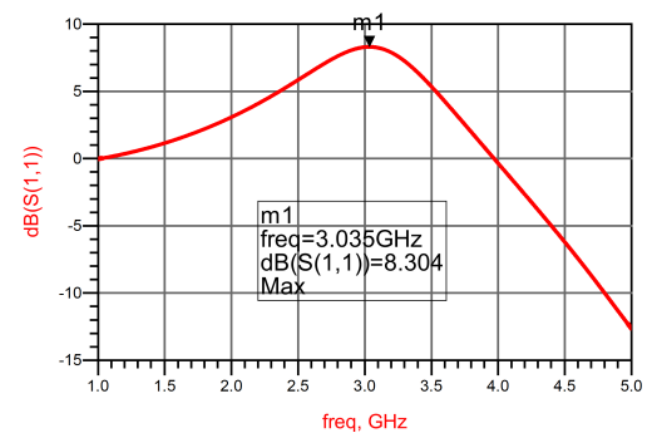

Figure 5. The port reflection coefficient.
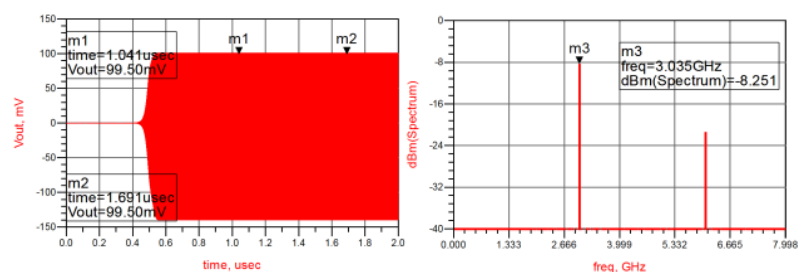

Figure 6. The transient simulation results in the time and frequency domain.

The transient simulation results in the time and frequency domain are shown in Fig. 6. It can be seen that the circuit can achieve a stable oscillation in a short time. 
The fundamental frequency of the output oscillation signal is $3.035 \mathrm{GHz}$ and the power is $-1.251 \mathrm{dBm}$ before attenuated by a $7 \mathrm{~dB}$ attenuator at the end of the circuit.

\subsection{Optimization with the virtual-ground method}

With the use of the negative resistance analysis method, the circuit has reached a steady oscillation. However, the negative resistance analysis method lacks an effective observation of the operating mechanism of the circuit. In order to solve the problem, the transmission analysis with a virtual-ground is used. And then the gain, the quality factor and other parameters of the circuit can be evaluated. Besides, based on the simulation results, the parameters of the designed VCO can be optimized to achieve a low phase noise VCO.

According to the virtual-ground analysis method, a new reference ground point should be firstly selected for analyzing the circuit conveniently without changing the signal transmission characteristics of the original VCO circuit in Fig. 4. Generally, the emitter electrode of the transistor is selected as the virtual-ground point [6] to separate the gain module and the tuning module explicitly. This is because the emitter electrode is in both the control circuit and the controlled circuit. Then the base electrode of the transistor can be chosen as the breakpoint in the circuit to analyze the characteristics of the oscillation [7]. The schematic diagram with a virtual-ground point at the emitter electrode is shown in Fig. 7.

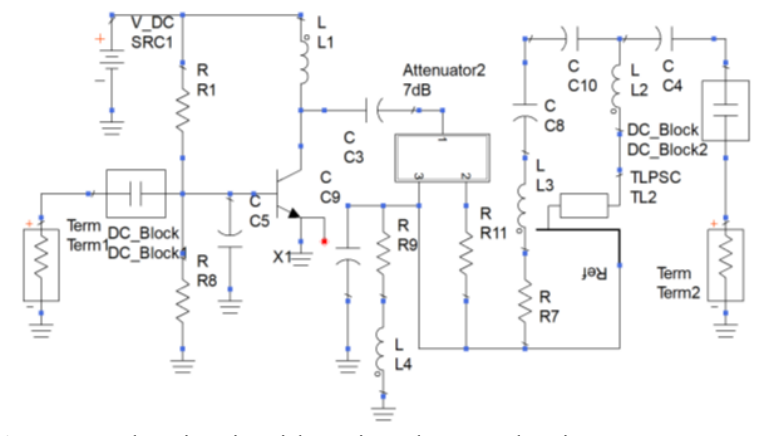

Figure 7. The circuit with a virtual-ground point

In order to achieve the maximum power transmission, the impedances of the two ports should be set as a pair of conjugate values according to the input impedance seen into the gain module. The circuit is simulated and the open-loop gain of the circuit can be calculated based on the formula in (7) [1].

$$
\text { Gain } \approx \frac{S_{21}}{1-S_{11} S_{22}}+S_{12}\left[\frac{\left(S_{21}-2\right) S_{21}-\left(1-S_{11} S_{22}\right)}{\left(1-S_{11} S_{22}\right)^{2}}\right]
$$

The simulation results are shown in Fig. 8. The curve of the open-loop gain is plotted in the polar coordinates as shown in Fig. 8(a). One net clockwise encirclement of the point $(1,0)$ is achieved as the frequency is increased, indicating that the Nyquist criterion can be met and the designed oscillator can start up [1]. Besides, curves of the magnitude and phase of the gain in (7) are plotted in the rectangular coordinates as shown in Fig. 8(b). Two zero phase crossings can be observed around the frequency of
$3.035 \mathrm{GHz}$, but only the first zero phase crossing occurs with negative slope and positive gain about $8.6 \mathrm{~dB}$ while the second occurs with negative gain, meaning that the closed-loop network will only oscillate at the first zero phase crossing which is $3.037 \mathrm{GHz}$. Though the simulated zero phase crossing is slightly higher than the desired operating frequency of $3.035 \mathrm{GHz}$, mainly caused by the difference between the virtual-ground analysis and the transient analysis, the analysis of the circuit functions and the parameter optimization are not affected. It can also be seen that, the zero phase crossing on the left present a steep slope, indicating that the circuit possess a high load quality factor. Based on the equation (8), the load quality factor at the crossing point is about 139 .

$$
Q=\frac{\omega}{2} \frac{\partial \varphi}{\partial \omega}
$$
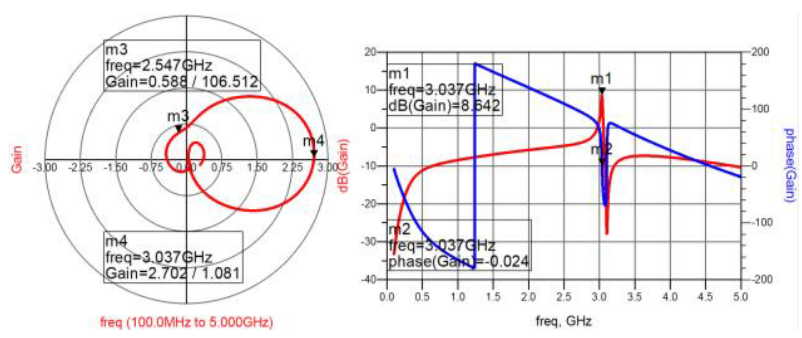

Figure 8. The curves of the gain in different coordinates: (a) the polar coordinates, (b) the rectangular coordinates.

\subsection{Harmonic balance simulation results}

The results of the harmonic balance simulation are shown in Fig. 9. It shows that the phase noises are $-71.87 \mathrm{dBc} / \mathrm{Hz}$ and $-85.123 \mathrm{dBc} / \mathrm{Hz}$ at the frequency offset of $300 \mathrm{~Hz}$ and $1 \mathrm{KHz}$, respectively, meeting the requirements of the ${ }^{85} \mathrm{Rb}$ atomic frequency standard well.

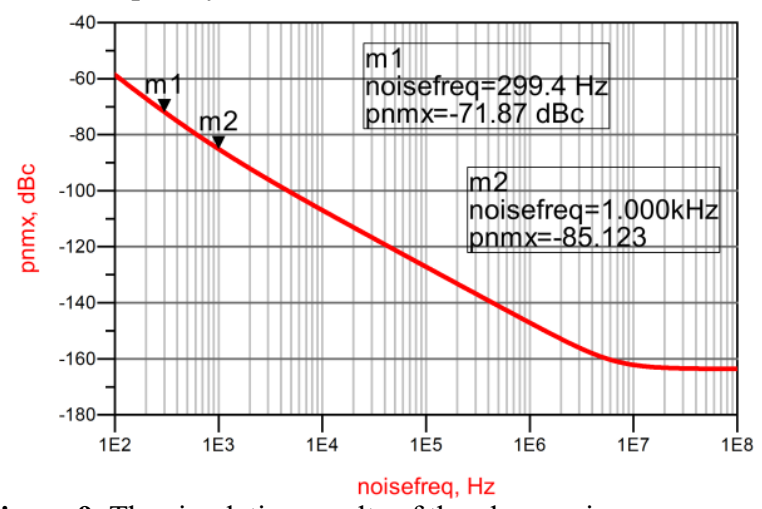

Figure 9. The simulation results of the phase noise.

\section{Test results}

In order to realize a VCO with a small size, 0402 SMT technology components are used and the circuit is laied out in a tightly enclosed topology. These choises resulted in a $10.5 \mathrm{~mm}$ by $8.8 \mathrm{~mm}$ by $0.6 \mathrm{~mm}$ final design which also includes the COAX and a grouned metal shield. The supply voltage of the VCO is $2.8 \mathrm{~V}$ dictated by that of the entire CSAC system which is $3.3 \mathrm{~V}$ and by the voltage regulator used to distribute it. The fabricated VCO board 
is shown in Fig. 10 with a size (the effective size) smaller than a dime.

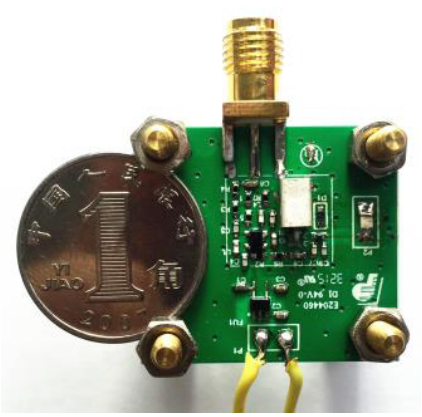

Figure 10. The fabricated VCO PCB.

When the VCO is tested with the same values of the passive components in Fig. 4, the test results are not in accordance with the simulation results well. Though the nonlinear simulation did not perfectly predict the VCO behaviours, it provided a good starting point to pursue optimizations. According to the simulation, performance of the designed VCO is mainly affected by capacitors of $\mathrm{C} 4, \mathrm{C} 5$ and $\mathrm{C} 6$ in Fig .4 when using the same COAX and varactor. Besides, the loaded factor quality of the $\mathrm{VCO}$ circuit can be augmented by increasing $\mathrm{C} 6$ or reducing $\mathrm{C} 4$, resulting in a lower phase noise. Doing so however, the output power will reduce and the VCO may not start up under all operating conditions. The test results show that the VCO can operate with better performance with an increased $\mathrm{C} 6$ and a reduced $\mathrm{C} 4$, this may benefit from the high transition frequency of the transistor used in the circuit. By some tuning on the values of the key capacitors described above, desired results were achieved. The test results show that the fundamental frequency is $3.035 \mathrm{GHz}$ when the varactor is reversely biased at $0.4 \mathrm{~V}$. The phase noises of the VCO are $-60.49 \mathrm{dBc} / \mathrm{Hz} @ 300 \mathrm{~Hz}$, $-73.08 \mathrm{dBc} / \mathrm{Hz} @ 1 \mathrm{KHz}$ and $-97.48 \mathrm{dBc} / \mathrm{Hz} @ 10 \mathrm{KHz}$ shown in Fig. 11. Moreover, the phase noise at the frequency offset of $10 \mathrm{KHz}$ is much better than $-90 \mathrm{dBc} / \mathrm{Hz} @ 10 \mathrm{KHz}$ claimed in the doctoral thesis [8].

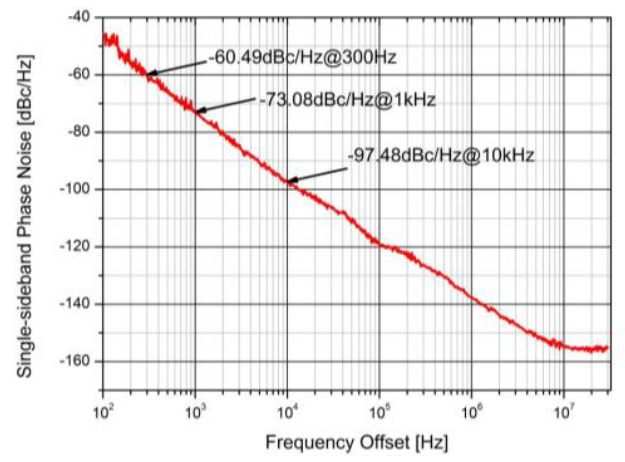

Figure 11. The test results of the phase noise.

The power of the fundamental signal is $-1.13 \mathrm{dBm}$ before attenuated by a $7 \mathrm{~dB}$ attenuator at the end of the circuit, meeting the requirement of the rubidium atomic clock based on the CPT phenomenon [8]. The tuning range of the reverse bias voltage of the varactor is $0 \sim 2.5 \mathrm{~V}$.
The voltage-controlled tuning curve is shown in Fig. 12, the tuning sensitivity is about $12 \mathrm{MHz} / \mathrm{V}$ when the tuning voltage changes between $0 \sim 2.0 \mathrm{~V}$.

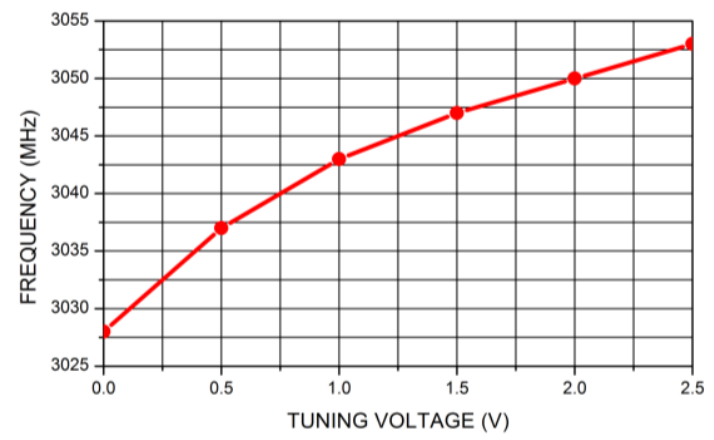

Figure 12. The voltage-controlled tuning curve of the VCO.

\section{Conclusions}

A small size and low phase noise $3.035 \mathrm{GHz}$ VCO based on a COAX with high quality factor is proposed. The circuit is designed, simulated and optimized by the combination of the negative-resistance analysis method and the virtual-ground technology. The test results show that the phase noises are $-60.49 \mathrm{dBc} / \mathrm{Hz}$ and $-97.48 \mathrm{dBc} / \mathrm{Hz}$ at the frequency offset of $300 \mathrm{~Hz}$ and $10 \mathrm{KHz}$ respectively, the power of the fundamental signal is $-1.13 \mathrm{dBm}$ and the voltage-controlled tuning sensitivity is about $12 \mathrm{MHz} / \mathrm{V}$, indicating that the designed $\mathrm{VCO}$ in this paper can meet the requirements of the ${ }^{85} \mathrm{Rb} \mathrm{CPT}$ atomic clocks well.

\section{References}

1. L. Hollberg, J. Kitching, L. Liew, et al, A chip-scale atomic clock based on ${ }^{87} \mathrm{Rb}$ with improved frequency stability, Optics Express, 13(4):1249-1253 (2005).

2. S. Knappe, V. Shah, P. D. D. Schwindt, et al, A microfabricated atomic clock, Applied Physics Letters, 85(9):1460-1462 (2004).

3. S. Romisch, R. Lutwak, Low-power, 4.6-GHz, Stable Oscillator for CSAC, International Frequency Control Symposium and Exposition, 448-451 (2006).

4. M. Randall, T. Hock, General oscillator characterization using linear open-loop S-parameters, Microwave Theory \& Techniques IEEE Transactions on, 49(6):1094-1100 (2001).

5. D. M. Pozar, Microwave Engineering, John Wiley and Sons, 613-627 (2012).

6. S. Alechno, Analysis method characterizes microwave oscillations, Oscillator Analysis, Part 1, Microwaves \& RF, 83-86 (1997).

7. A. Brannon, M. Jankovic, J. Breitbarth, et al, A Local Oscillator for Chip-Scale Atomic Clocks at NIST, International Frequency Control Symposium and Exposition, 443-447 (2006).

8. K. Deng, Experimental Study on the Miniaturized Coherent Population Trapping Atomic Clock, Ph.D. Thesis of Peking University (2011). 